
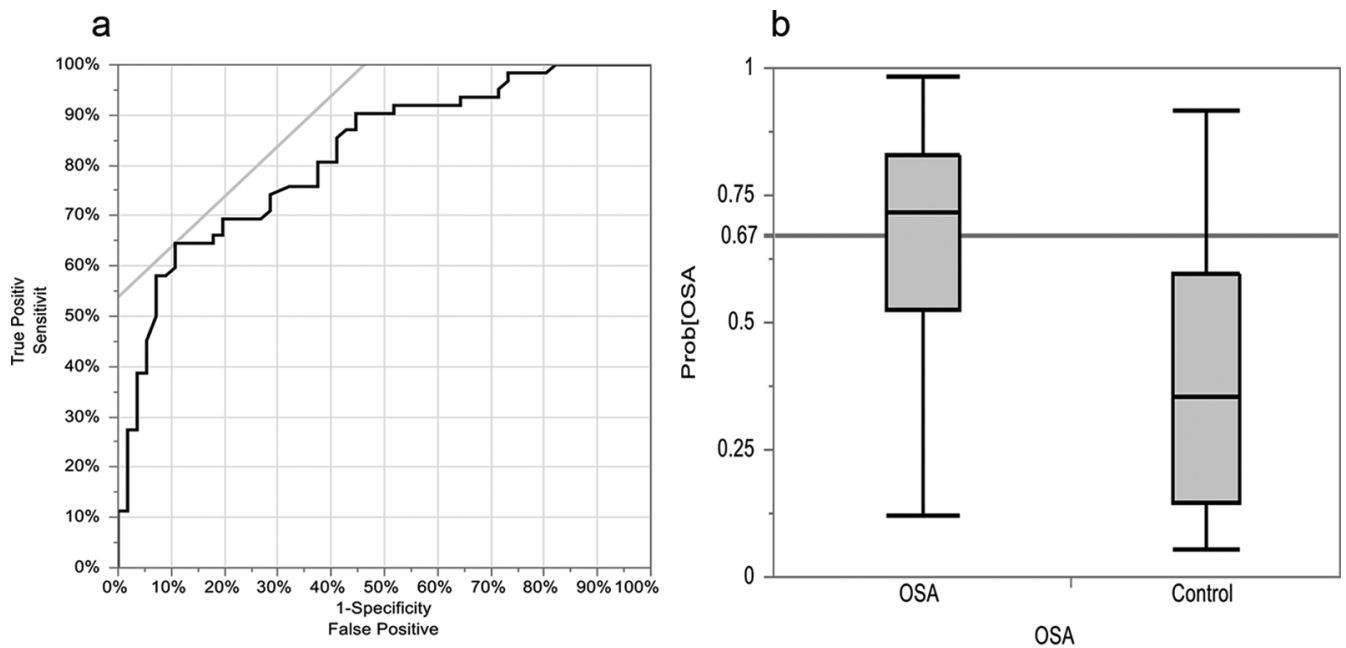

C
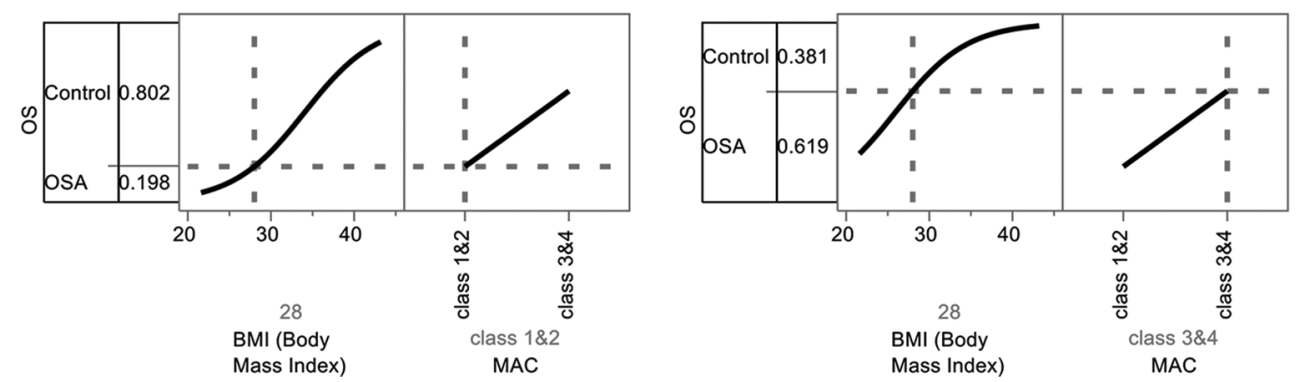

Abstract P019 Figure 2 a) Receiver operating characteristic (ROC) curve for backward logistic regression model for surface and clinical variables with area under the curve (AUC) of 0.82 . b) Box plot showing logistic regression model score for OSA subjects and controls. A cut-off value of 0.67 (red line, b) produced $65 \%$ sensitivity and $89 \%$ specificity for identification of OSA patients with an AHI $\geq 5$ events/hour. c) Prediction profilers for logistics multiple regression - surface and clinical predictors (backward model). Prediction formula: Prob (OSA) $=1 /(1+$ Exp $(-((-7.88)+0.23 * B M I+$ $1.88 * \mathrm{MAC}))$ )

Mallampati airway classification, sleep apnoea clinical score, Epworth sleepiness scale and 3-D stereophotography for surface craniofacial analysis (figure1).

Results Surface craniofacial risk factors (phenotype) were identified for OSA Caucasian males, with the predominant characteristics being: an enlarged neck circumference $(\mathrm{p}<0.001)$, short neck $(\mathrm{p}<0.001)$, large mandibular width $(\mathrm{p}<0.001)$, forward head posture $(\mathrm{p}<0.001)$ and increased lower anterior facial height $(\mathrm{P}<0.002)$. Multiple regression analysis for the surface predictors, both with and without clinical variables, identified a range of prediction models with moderate to high sensitivity and specificity, with an area under the receiver operating characteristics curve (AUC) between 0.73-0.82. A higher positive and lower negative likelihood-ratios were identified for the combination model of the surface and clinical variables $(L R+6.02, L R-0.40$ respectively) when compared to the surface model alone. A high positive post-test probability and odds ratio $(87 \%$, $\mathrm{OR}=6.8$, respectively) were also identified in the combination model. The surface model, with and without clinical variables, not only successfully identified OSA subjects from controls (AUC $=0.77,0.82$ respectively) but also presented as a marker (figure 2).

Conclusion This case-control study demonstrated the existence of a surface craniofacial phenotypic pattern, identified a predictive model and marker for OSA in Caucasian males, using 3D-surface imaging analysis and clinical tools.

\section{P020 AN AUDIT OF SLEEP STUDY RESULTS IN PATIENTS UNDERGOING PRE-OPERATIVE ASSESSMENT}

Vicky Cooper*. Salford Royal NHS Foundation Trust, Salford

10.1136/bmjresp-2019-bssconf.20

Introduction Undiagnosed obstructive sleep apnoea (OSA) presents a significant risk to patients undergoing general anaesthesia. The STOP-BANG questionnaire is a useful screening tool for predicting a high probability of OSA. This study examines the results of the pre-operative screening at Salford Royal NHS Foundation Trust.

Methods The sleep study results from 857 patients (658 male) aged 20-87 years $(57.1 \pm 0.4$ years) who underwent a preoperative assessment and scored $\geq 3$ on the STOP-BANG questionnaire were retrospectively analysed. Patients were categorised as have no, mild, moderate or severe OSA according to their Apnoea Hypopnoea Index (AHI) $(n=675)$ or Oxygen Desaturation Index (ODI) $(n=182)$. using AASM criteria b (4\% desaturation).

The effects of gender, age, body mass index (BMI), neck circumference (NC) and Epworth Sleepiness Score (ESS) were independently assessed and differences between groups were compared using One Way ANOVA. Results are reported as mean \pm standard error.

Results The number of patients having no, mild, moderate or severe OSA were 215 (25\%), 250 (29\%), 214 (25\%) and 178 (21\%), respectively. 
The proportion of males increased as severity increased. The percentage of males who had no, mild, moderate or severe OSA was $64.6 \%, 72.0 \%, 86.0$ and $87.0 \%$, respectively.

Mean age was significantly lower $(\mathrm{P}<0.001)$ in the no OSA compared to all 3 OSA categories. Those with moderate OSA were also significantly older $(\mathrm{P}<0.05)$ than those with mild OSA.

Those with severe OSA had a significantly higher average BMI compared to all other categories $(\mathrm{P}<0.05)$.

Average neck circumference significantly increased with severity of OSA.

There was no significant difference in average ESS between groups.

Conclusion Seventy-five percent of pre-operative patients with a STOP-BANG score $\geq 3$ had some degree of OSA. The severity of OSA was dependent on gender, age, BMI and neck circumference. ESS was not helpful in predicting presence or severity of OSA.

\section{P021 NON-INVASIVE VENTILATION FOR OBESE PATIENTS WITH OSA FAILING REGULAR CPAP: A PROSPECTIVE OBSERVATIONAL COHORT STUDY}

\footnotetext{
${ }^{1,2}$ Athanasius Ishak*, ${ }^{2}$ Michelle Ramsay, ${ }^{1,2}$ Nicholas Hart, ${ }^{1,2}$ Joerg Steier. ${ }^{1}$ King's College London Faculty of Life Sciences and Medicine, Centre for Human and Applied Physiological Science (CHAPS), London, UK; ' 'Guy's and St Thomas' NHS Foundation Trust Lane Fox Respiratory Unit, London, UK
}

\subsection{6/bmiresp-2019-bssconf.21}

Introduction Continuous positive airway pressure (CPAP) therapy is the standard treatment for obstructive sleep apnoea (OSA), however, patients frequently have sub-optimal longterm adherence. We hypothesised that bilevel positive airway pressure (BPAP) therapy may improve adherence and outcomes in patients who cannot tolerate CPAP.

Methods Patients with OSA who had sub-optimal CPAP usage ( $<4$ hours/day) and were referred to our sleep centre between 2014-2017 for BPAP were included. Age, gender, body-massindex (BMI), co-morbidities, CPAP use and reasons for failure, Epworth Sleepiness Score (ESS), sleep study data, spirometry data and average maximum nightly compliance were recorded.

Results We included 52 patients with OSA who required CPAP $>15 \mathrm{cmH} 20$ (71\% male, age 58 (15) years, BMI 42.6 (10.1) kg/m2, AHI 51.1 (30.4)/hour); 62\% had respiratory co-morbidities affecting nocturnal breathing (COPD, OHS). CPAP was used for 199 (106-477) days prior to referral. Reasons for CPAP failure were intolerance of pressures (23\%), uncontrolled symptoms (23\%), mask problems (21\%), adverse effects (13\%), claustrophobia (8\%), co-morbidities $(8 \%)$, and other issues (4\%). Lower expiratory positive airway pressures (EPAP) were needed to control nocturnal breathing compared to CPAP (10 (8-12) vs 16.8 (15.7-19.2) $\mathrm{cmH} 20, \mathrm{p}=0.001)$ and patients achieved better adherence (7.0 (4.0-8.5) vs $2.5(1.6-6.7)$ hours/night, $\mathrm{p}=0.028)$ and better symptom control (ESS 4.0 (1.0-7.0) vs 10.0 (6.0$17.0)$ points, $\mathrm{p}=0.039$ ) on BPAP.

Discussion In patients with OSA with limited success on CPAP therapy, BPAP is better tolerated and achieves sufficient respiratory and symptom control.

\section{P022 \\ AN INVESTIGATION INTO THE EFFECTS OF A 20- MINUTE NAP-OPPORTUNITY ON COGNITIVE FUNCTION IN STUDENTS AGED 18-30}

${ }^{1}$ Suraiya Haddad, ${ }^{1}$ Rezwana Rahman, ${ }^{1}$ Felyx Wong ${ }^{*},{ }^{1}$ W Jackson, ${ }^{1,2}$ Mary Morrell. ${ }^{1}$ Imperial College London, London, UKi ${ }^{2}$ Academic Unit of Sleep and Ventilation, National Heart and Lung Institute, Imperial College London, Royal Brompton and Harefield National Health Service Foundation Trust

\subsection{6/bmjresp-2019-bssconf.22}

Introduction Sleep-deprivation is increasing in prevalence and compensatory napping is becoming common practice. Little is known about the cognitive benefits derived from a nap in sleep-deprived individuals, or within the student population. Furthermore, few studies have investigated the cognitive benefits of napping using the incongruent Stroop test. This study aimed to investigate the effects of a nap-opportunity on cognitive function in individuals aged 18-30.

Methods A randomised, crossover study was conducted. Participants were recruited from Imperial College London and were allocated to either control (no nap-opportunity) or intervention (20-minute nap-opportunity) groups.

The incongruent Stroop test was used to measure reaction time (RT) and percentage accuracy, which were used as markers of cognitive function. $\Delta \mathrm{RT}$ and $\Delta$ percentage accuracy, between control and nap-opportunity groups were compared. Paired and unpaired t-tests were used for the analysis of RT. Wilcoxon signed-rank and Mann-Whitney-U tests were used to test the percentage accuracy. The study was approved by the Imperial College Medical Education Ethics Committee.

Results Participants $(n=29)$ had a mean age of $20(S D \pm 1.4)$ years, $55 \%$ male. In those who were acutely sleep-deprived, the RT improved in the intervention group following a 20 minute nap-opportunity, compared to the control group $(\mathrm{p}=0.01$; figure 1$)$. Overall, no significant difference in $\Delta$

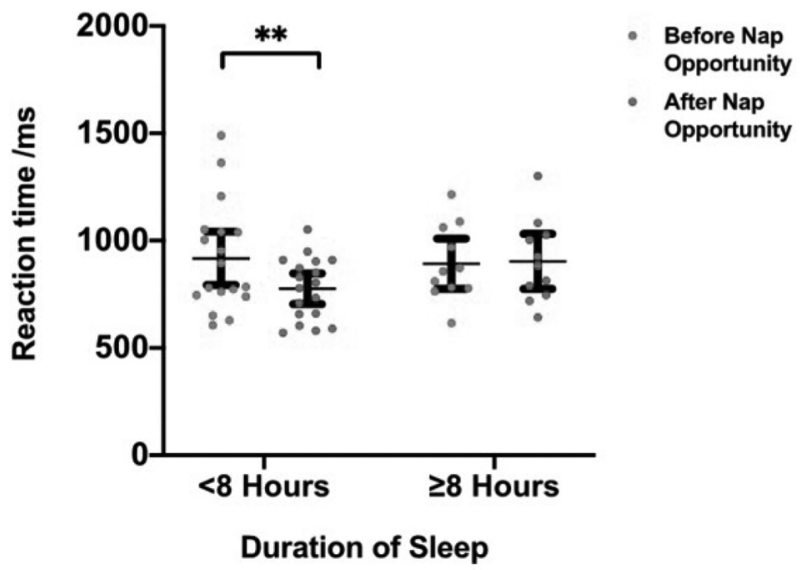

Abstract P022 Figure 1 Dot plot showing reaction times in incongruent Stroop test, measured before and after a 20 minute napopportunity in individuals who slept for $<8 \mathrm{hrs}$ the night before the test $(n=18)$ and $=8 \mathrm{hrs}$ the night before the test $(n=11)$. Data presented as mean $\pm 95 \% \mathrm{Cl}$. Paired t-test was used to compare reaction times before and after nap-opportunity. Unpaired t-tests were used to compare baselines and endpoints of the two groups. Significant change in reaction time was observed following nap-opportunity in individuals who slept $<8$ Hours before the test day $(p=0.01275){ }^{*}=P<0.05$,

${ }^{* *}=\mathrm{P}<0.01,{ }^{* * *}=\mathrm{P}<0.001$ 